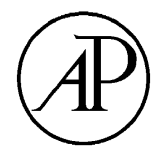

ACADEMIC PRESS
Available online at www.sciencedirect.com

science@directo

Theoretical Population Biology 64 (2003) 331-343
Theoretical

Population

Biology

http://www.elsevier.com/locate/ytpbi

\title{
The age-structured lottery model
}

\author{
Sonya Dewi ${ }^{1}$ and Peter Chesson* \\ Ecosystem Dynamics Group, Australian National University, Canberra, Australia
}

Received 6 May 2003

\begin{abstract}
The lottery model of competition between species in a variable environmental has been influential in understanding how coexistence may result from interactions between fluctuating environmental and competitive factors. Of most importance, it has led to the concept of the storage effect as a mechanism of species coexistence. Interactions between environment and competition in the lottery model stem from the life-history assumption that environmental variation and competition affect recruitment to the adult population, but not adult survival. The strong role of life-history attributes in this coexistence mechanism implies that its robustness should be checked for a variety of life-history scenarios. Here, age structure is added to the adult population, and the results are compared with the original lottery model. This investigation uses recently developed shape characteristics for mortality and fecundity schedules to quantify the effects of age structure on the long-term low-density growth rate of a species in competition with its competitor when applying the standard invasibility coexistence criterion. Coexistence conditions are found to be affected to a small degree by the presence of age structure in the adult population: Type III mortality broadens coexistence conditions, and type I mortality makes them narrower. The rates of recovery from low density for coexisting species, and the rates of competitive exclusion in other cases, are modified to a greater degree by age structure. The absolute rates of recovery or decline of a species from low density are increased by type I mortality or early peak reproduction, but reduced by type III mortality or late peak reproduction. Analytical approximations show how the most important effects can be considered as simple modifications of the long-term lowdensity growth rates for the original lottery model.
\end{abstract}

(C) 2003 Elsevier Inc. All rights reserved.

Keywords: Life history; Lottery model; Invasibility analysis; $\Delta$-Measure; Storage effect; Mortality schedule; Fecundity schedule

\section{Introduction}

In the study of species coexistence in a variable environment, the lottery model (Chesson and Warner, 1981) has had a pivotal role. In particular, it has shown that species coexistence results from interactions between the direct responses of species to environmental variation and their responses to competition. Lifehistory characteristics have an important role in creating such interactions (Chesson, 1989, 1994). For example, in the lottery model, iteroparity of at least some of the interacting species is essential to coexistence. It is

\footnotetext{
${ }^{*}$ Corresponding author. Present address: Section of Evolution and Ecology, Division of Biological Sciences, University of California, Shields Avenue, 3331 Storer Hall, Davis, CA 95616, USA. Fax: + 1530-752-1449.

E-mail addresses: s.dewi@cgiar.org (S. Dewi),plchesson@ucdavis. edu (P. Chesson).

${ }^{1}$ Present address: Center for International Forestry Research, Bogor, Indonesia 16680.
}

important also that environmental variation affect reproduction more strongly than adult survival (Chesson, 1990). In different models, different life-history characteristics may be key, but the roles displayed by the life-history characteristics of the lottery model are retained (Chesson, 1990). Synthesis of the key features of coexistence in the lottery and related models led to the concept of the storage effect (Warner and Chesson, 1985) as a general mechanism of species coexistence that results from interactions between responses to environment and competition. These interactions are created by life-history traits that allow population increases but minimize population losses. For example, persistent stages in the life cycle, such as dormant seeds or longlived adults, may increase in numbers during favorable periods, but have low mortality rates under most conditions, ensuring that gains during favorable times are not lost immediately unfavorable times return. However, in spite of the important role of life histories in species coexistence, the original lottery model allows 
only very simply life histories to be represented because the only population structure that it recognizes is the distinction between juveniles and adults. The adult population has no structure, and for that reason, we refer to the original model as the nonstructured lottery model, or NLM. In this manuscript, we introduce the structured lottery model, or SLM, in which we investigate the effects on species coexistence of age structure in the adult population.

In order to characterize age structure, in Dewi and Chesson (2003) we introduced shape characteristics, that we term $\Delta$-measures, as tools for summarising mortality and fecundity schedules. These measures prove especially useful here. In order to compare the NLM and SLM, several constraints must be imposed to ensure that any differences in the behavior of the two models are due to inherent characteristics of the models. It is useful to remember that the SLM is the general case, and the NLM is a special case of the SLM where mortality and fecundity are independent of age.

The behavior of the NLM is determined by two lifehistory characteristics: (i) the expected lifetime $(=1 / \tilde{\delta}$, where $\tilde{\delta}$ is the age-independent death rate), and (ii) a temporally fluctuating but age-independent birth rate (Chesson, 1989, 1994). In our investigation of the SLM, the values of life-history parameters will be constrained to achieve an expected lifetime and fluctuating agedependent birth rate equivalent to those in the NLM while allowing mortality and fecundity rates to be agedependent.

To study species coexistence, we use invasibility analysis (Chesson and Ellner, 1989; Ellner, 1989), and thus focus on the long-term population growth rate of one species (the invader), which is held at low density, in competition with another species (the resident) that has achieved its stationary distribution of fluctuations over time. In studying species coexistence in the SLM, we are particularly concerned with identifying the sorts of situations where age structure can be ignored, or when some simple summary of the nature of age structure suffices to characterize its important effects. In general, having to account for age structure greatly complicates theoretical and empirical investigations in ecology. Thus, it is important to be able delineate the kinds of situations where accounting for age structure is essential, and to account for it economically in those situations where it cannot be ignored.

\section{The model}

To generalize the NLM to the age-structured case, we assume that the probability of survival of an individual of species $i$ from age $x$ to age $x+1$ is $\delta_{i x}$, and that the number of offspring produced by an individual of species $i$ and age $x$ in the time interval $t$ to $t+1$ is $b_{i}(t) k_{i x}$, i.e. the product of a quantity $b_{i}(t)$ that varies with time, and a quantity $k_{i x}$ that varies with age. Both of these quantities are species-specific also. This model of reproduction assumes that all age classes respond similarly to variation in their common temporally varying environment, but that different ages do have different average reproductive outputs. We refer to the quantity $k_{i x}$ as the age-specific modulation of reproduction, and subject it to the constraint

$\frac{\sum_{x=1}^{\infty} k_{i x} l_{x}}{\sum_{x=1}^{\infty} l_{x}}=1$,

where $l_{x}$ has the usual meaning as the probability of surviving to age $x$, and is determined by the $\delta_{i x}$. Constraint 1 is necessary to prevent overparameterization of the model. In effect, it restricts the average of the $k_{i x}$ weighted by the probability of surviving to age $x$. This constraint relates to the stationary age structure of the population, i.e. the age structure that it would have when its total population size is constant, and its age structure has stabililized. As the stationary age distribution is proportional to $l_{x}$, the weighted average (1) is an average over the stationary age distribution of the population. Note that the denominator in (1) is equal to the expected adult longevity, and that its reciprocal, which we denote by $\tilde{\delta}$, is the value of $\delta_{i x}$ that would apply in an age-independent population with the same longevity. We refer to it here as the age-independent death rate.

The total density of offspring for a given species $i$ produced in one unit of time is the sum over all adult age classes $x$ of $P_{i x} b_{i}(t) k_{i x}$, where $P_{i x}$ is defined as the fraction of the total space in the system occupied by species $i$, age $x$. In the SLM, the offspring of any species are assumed to compete with the offspring of all species for settling sites that are given up by adult deaths. The total density of these sites is the sum over species and adult age classes of $P_{i x} \delta_{i x}$. Competition for these sites is assumed to be by lottery: the probability that a given species gains a site is simply proportional to the density of its offspring in the system.

Defining $P_{i .}(t)$ to be the total density of species $i$ in the system, i.e. the sum over all age classes of $P_{i x}$, the various elements of the model lead to the following equation for species $i$ in competition with just one other species, $j$ :

$$
\begin{aligned}
& P_{i .}(t+1)=\left(\sum_{x=1}^{\infty}\left(1-\delta_{i x}\right) P_{i x}(t)\right) \\
& \quad+\left(\sum_{x=1}^{\infty} \delta_{i x} P_{i x}(t)+\delta_{j x} P_{j x}(t)\right) \\
& \quad \times\left(\frac{b_{i}(t) \sum_{x=1}^{\infty} k_{i x} P_{i x}(t)}{b_{i}(t) \sum_{x=1}^{\infty} k_{i x} P_{i x}(t)+b_{j}(t) \sum_{x=1}^{\infty} k_{j x} P_{j x}(t)}\right) .
\end{aligned}
$$


The first part of this equation is simply adult survival, and the second part is the product of the density of space becoming available by adult death and the proportion of this space that is allocated to species $i$ according to its representation of offspring in the total offspring pool.

\section{Analysis}

For invasibility analysis, in a two-species setting, we first of all need to understand the dynamics of a singlespecies resident $(j)$, and then the dynamics of an invader $(i)$, whose density is effectively zero, in competition with this resident. Because all space becomes filled at the end of each reproductive cycle, a single-species resident simply has $P_{j .}=1$, for all $t$, and therefore has a growth rate of zero. This single-species resident simply captures all available space and the total number of new recruits (new individuals entering the adult population) equals the total number of adult deaths in one unit of time, viz the sum over $x$ of $P_{i x} \delta_{i x}$. Thus, the age-structured dynamics of the resident population can be represented by the following Lefkovitch matrix model:

$$
\left(\begin{array}{c}
P_{j 1} \\
P_{j 2} \\
P_{j 3} \\
\vdots \\
P_{j s}
\end{array}\right)(t+1)=\left(\begin{array}{cccc}
\delta_{j 1} & \delta_{j 2} & \delta_{j 3} & \cdots \\
\left(1-\delta_{j 1}\right) & 0 & 0 & \cdots \\
0 & \left(1-\delta_{j 2}\right) & 0 & \cdots \\
\vdots & \vdots & \ddots & \cdots \\
0 & 0 & \cdots & \left(1-\delta_{j, s-1}\right)
\end{array}\right.
$$

The first row of this matrix has a novel interpretation. Rather than representing the fecundity of an age class, it is mortality because each age class contributes to recruitment by making space available through death. In spite of fluctuations in birth rates modeled by the temporally varying quantity $b_{j}(t)$, recruitment does not fluctuate because birth rate fluctuations are countered by equivalent fluctuations in competition (a reflection of covariance between environment and competition, discussed in Chesson, 1989, 1994). Thus, in spite of the fact that underlying Eq. (3) are stochastic nonlinear processes, the dynamics of age structure are linear and deterministic. The novel interpretation of the first row of the matrix, however, is a reflection of these underlying nonlinear processes.

The above matrix model satisfies the condition for convergence to a stable age distribution according to the Perron-Frobenius theorem (see, e.g., Caswell, 2001). Therefore, the resident converges on its stable age distribution for which the ratio $P_{j x} / P_{j}=u_{j x}$ does not fluctuate over time. Moreover, as the resident's growth rate is zero, the stable age distribution is the stationary age distribution given by the equation

$u_{j x}=\frac{l_{j x}}{\sum_{x=1}^{\infty} l_{j x}}$.

As a consequence of convergence on the stationary age distribution, the average of the death rate over all individuals in the resident population, $\hat{\delta}_{j}(t)$, does not fluctuate over time and is equal to the age-independent death rate corresponding to its mortality schedule, and is given by the equation

$$
\begin{aligned}
\hat{\delta}_{j}=\sum_{x=1}^{\infty} \delta_{j x} u_{j x} & =\frac{\sum_{x=1}^{\infty} \delta_{j x} l_{j x}}{\sum_{x=1}^{\infty} l_{j x}} \\
& =\frac{1}{\sum_{x=1}^{\infty} l_{j x}} \\
& =\tilde{\delta}_{j},
\end{aligned}
$$

where the last equation defines $\tilde{\delta}_{j}$. Note: the derivation uses the identity $\sum_{x=1}^{\infty} \delta_{j x} l_{j x}=1$, which is true because $\delta_{j x} l_{j x}$ is the probability distribution for any given individual's age of death.

As a consequence also of the stationary age distribution, the average modulation of reproduction

$$
\left.\begin{array}{c}
\delta_{j s} \\
0 \\
0 \\
\vdots \\
\left(1-\delta_{j s}\right)
\end{array}\right)\left(\begin{array}{c}
P_{j 1} \\
P_{j 2} \\
P_{j 3} \\
\vdots \\
P_{j s}
\end{array}\right)(t)
$$

of the resident is given by the equation

$$
\begin{aligned}
\hat{k_{j}} & =\sum_{x=1}^{\infty} k_{j x} u_{j x} \\
& =\frac{\sum_{x=1}^{\infty} k_{j x} l_{j x}}{\sum_{x=1}^{\infty} l_{j x}} \\
& =\tilde{k_{j}} .
\end{aligned}
$$

However, applying constraint (1), which eliminates parameter redundancy, we see that modulation of reproduction for the resident reduces to

$\hat{k_{j}}=\tilde{k_{j}}=1$.

For the invader, the key dynamical equation (2) can be written as

$$
\begin{aligned}
P_{i .}(t+1)= & P_{i .}(t)\left\{\sum_{x=1}^{\infty}\left(1-\delta_{i x} u_{i x}(t)\right)\right. \\
& \left.+\sum_{x=1}^{\infty} \delta_{j x} u_{j x} \frac{b_{i}(t) \sum_{x=1}^{\infty} k_{i x} u_{i x}(t)}{b_{j}(t) \sum_{x=1}^{\infty} k_{j x} u_{j x}(t)}\right\},
\end{aligned}
$$


where $u_{i x}(t)=P_{i x}(t) / P_{i .}(t)$ and $u_{j x}(t)=P_{j x}(t) / P_{j .}(t)$, the age structures of invader and resident.

By substituting Eqs. (4) and (5) into Eq. (6), we obtain

$$
\begin{aligned}
P_{i .}(t+1)= & P_{i .}(t)\left\{\sum_{x=1}^{\infty}\left(1-\delta_{i x} u_{i x}(t)\right)\right. \\
& \left.+\tilde{\delta}_{j} \frac{b_{i}(t) \sum_{x=1}^{\infty} k_{i x} u_{i x}(t)}{b_{j}(t)}\right\} .
\end{aligned}
$$

From Eq. (7), we see that the mortality schedule of the resident does not affect population dynamics of the invader at all. It is only the age-independent death rate of the resident $\left(\tilde{\delta}_{j}\right)$ that matters. Since Eq. (7) does not involve density of the resident in any form, we can express Eq. (7) in matrix form as
$\Delta_{f}^{(1)}=\sum_{x=1}^{\infty} x \tilde{\delta} l_{x}\left(k_{x}-1\right)$

where $\tilde{\delta}=1 / \sum_{x} l_{x}$ is the age-independent death rate.

The quantities $\Delta_{m}$ and $\Delta_{f}$ measure departure of the given mortality and fecundity schedules from the corresponding age-independent mortality and fecundity schedules. Given the usual three way classification of mortality into types I-III (Roff, 1992), $\Delta_{m}$, has the following property:

$\Delta_{m}\left\{\begin{array}{l}>0 \text { for type I: mortality increases with age, } \\ =0 \text { for type II: age-independent mortality, } \\ <0 \text { for type III: mortality decreases with age. }\end{array}\right.$

$$
\left(\begin{array}{c}
P_{i 1} \\
P_{i 2} \\
P_{i 3} \\
\vdots \\
P_{i s}
\end{array}\right)(t+1)=\left(\begin{array}{ccccc}
\tilde{\delta}_{j} k_{i 1} \rho(t) & \tilde{\delta}_{j} k_{i 2} \rho(t) & \tilde{\delta}_{j} k_{i 3} \rho(t) & \ldots & \tilde{\delta}_{j} k_{i s} \rho(t) \\
\left(1-\delta_{i 1}\right) & 0 & 0 & \ldots & 0 \\
0 & \left(1-\delta_{i 2}\right) & 0 & \ldots & 0 \\
\vdots & \vdots & \ddots & \ldots & \vdots \\
0 & 0 & \ldots & \left(1-\delta_{i, s-1}\right) & \left(1-\delta_{i s}\right)
\end{array}\right)\left(\begin{array}{c}
P_{i 1} \\
P_{i 2} \\
P_{i 3} \\
\vdots \\
P_{i s}
\end{array}\right)(t)
$$

where $\rho(t)=b_{i}(t) / b_{j}(t)$.

The dynamics of the invader represented by the equation above follow a Lefkovitch matrix with temporally variable recruitment rates, and techniques for such matrices can be used. Since the interest is in determining conditions for coexistence, we can use an approach developed recently for considering the effects of age structure for average values of the population growth rate near zero (Dewi and Chesson, 2003). We briefly summarize this approach below.

\subsection{Overview of $\triangle$-measures, $N L M$ and the storage effect}

\subsection{1. $\Delta$-measures}

Statistical characteristics of age-specific mortality and fecundity schedules, which we term $\Delta$-measures, are introduced in Dewi and Chesson (2003), and used to study density-independent population growth. We provide here a minimal summary needed for the analysis of the SLM.

Given any mortality, $\delta_{x}$, and fecundity schedules, $b_{x}$, where $x$ ranges from 1 to $\infty$, we define

$$
\Delta_{m}^{(1)}=\sum_{x=1}^{\infty} x l_{x}\left(\delta_{x}-\tilde{\delta}\right)
$$

This measure thus gives a signed quantification of departure from type II or age-independent mortality. These different mortality-schedule types are illustrated in Fig. 1 in piecewise linear forms, which we use in our simulations.

Similar to $\Delta_{m}, \Delta_{f}$ gives a signed quantification of departure from age-independent reproduction. Fundamentally, $\Delta_{f}$ is positive if higher age-specific reproductive rates occur at younger ages, negative if these rates are higher at older ages, and zero if reproduction does not vary with age. In terms of the classification of

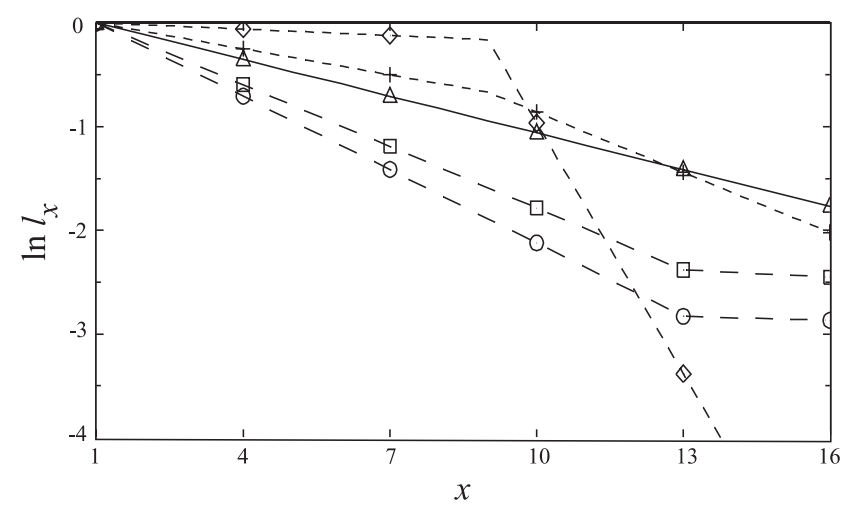

Fig. 1. Mortality schedules of type I (short dashes) with $\Delta_{m}=$ $0.2025(+), \Delta_{m}=0.4086(\diamond)$, of type II $\left(\Delta_{m}=0\right)$ (solid, $\triangle$ ) and of type III (long dashes) with $\Delta_{m}=-4.1589(\circ), \Delta_{m}=-1.9485(\square)$. 


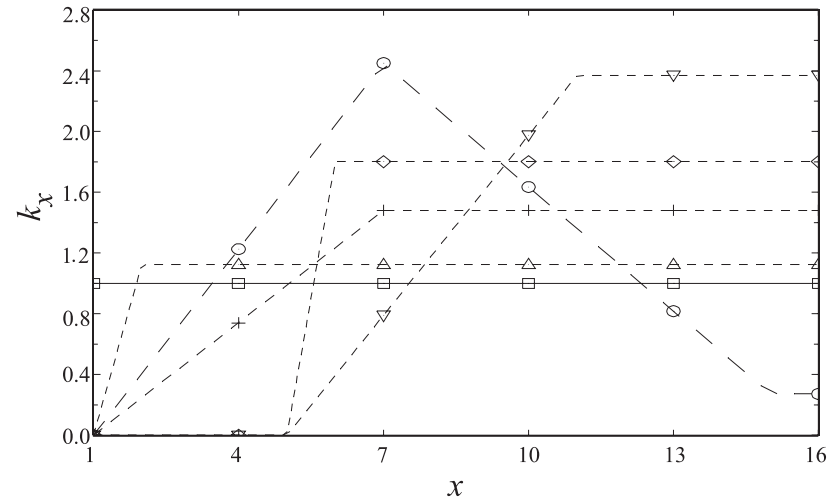

Fig. 2. Age-dependent modulation of reproduction $\left(k_{x}\right)$ for early peak reproduction (long dashes) with $\Delta_{f}=-1.2545(\circ)$, age-independent reproduction $(\square)$, and delayed peak reproduction (dashes) with $\Delta_{f}=1(\triangle), \Delta_{f}=3.1594(+), \Delta_{f}=5(\diamond)$, and $\Delta_{f}=7.1594(\nabla)$, when combined with a type II mortality schedule.

Roff (1992), we have

$\Delta_{f}\left\{\begin{array}{l}>0 \text { for delayed peak reproduction, } \\ =0 \text { for age-independent reproduction, } \\ <0 \text { for early peak reproduction. }\end{array}\right.$

In the numerical and simulation studies reported here, piecewise linear fecundity schedules are used, as illustrated in Fig. 2 plotting not $b_{x}$ but the modulation of fecundity $k_{x}$. These curves have the following characteristics:

- Age-independent reproduction with $k_{x}=1$ for all $x$.

- Uniform fecundity, i.e., a juvenile period, with zero fecundity, followed by age-independent reproduction after maturity: $k_{x}=0$ for $x<m$, and $k_{x}=k_{m}$ for $x \geqslant m$, where $m$ is age of maturity.

- Asymptotic fecundity, i.e., an increasing $k_{x}$ after the age of maturity and constant $k_{x}$ after a maximum value is reached.

- Triangular fecundity, which is similar to the asymptotic schedule except that after the maximum value is attained, $k_{x}$ declines until a certain age, after which it remains constant. Semelparity is a special case of the triangular schedule in which the peak is reached at the age of maturity and $k_{x}$ is zero in all subsequent age classes. However, since our matrix approach for calculating the population growth rate as the real dominant eigenvalue requires primitivity for the Perron-Frobenius theorem to apply (see for example Caswell, 2001) we do not use semelparous fecundity schedules in our study of the SLM. Nevertheless, semelparity does help illustrate the behavior of $\Delta_{f}$.

Being single numbers, $\Delta_{m}$ and $\Delta_{f}$ cannot hope to fully characterize departure from age independence. They are first order measures useful for first-order approximation of age dependence, which we use here. Higher-order measures, which jointly fully characterize mortality and fecundity schedules, and which might be used with higher order approximation, are given in Dewi and Chesson (2003).

These measures allow approximation of the long-term growth rate for a density-independent age-structured population. If the total population size is $N(t)$ at time $t$, the long-term growth rate, $\bar{r}$, is defined as follows:

$\bar{r}=\lim _{T \rightarrow \infty} \frac{\ln N(T)-\ln N(0)}{T}$.

Now assume that the mortality schedule for this population is $\delta_{x}$ and that age-dependent fecundity is of the form $\tilde{b}(t) k_{x}$, as defined for each individual species in the SLM. However, here there is only one species and reproduction is not followed by competition because we assume density independence. The temporal component of birth rate variation $\tilde{b}(t)$ is assumed to fluctuate over time with $E[\ln \tilde{b}(t)]-\ln \tilde{\delta}=\mu$ and $\operatorname{Var}(\ln \tilde{b}(t))=\sigma^{2}$. Different times are assumed statistically independent. Then, assuming that $\mu=O\left(\sigma^{2}\right)$, i.e. that the mean $\ln$ birth rate is same order as the temporal variance of the ln birth rate, and that the average mortality rate, $\tilde{\delta}$, is small, the long-term growth rate, $\bar{r}$, is shown in Dewi and Chesson (2003) to be

$\bar{r}=\frac{\tilde{\delta} \mu+\frac{1}{2} \tilde{\delta}(1-\tilde{\delta}) \sigma^{2}}{1+\tilde{\delta}\left(\Delta_{f}-\Delta_{m}\right)}+o\left(\sigma^{2}\right)$

The assumption that $\mu=O\left(\sigma^{2}\right)$ ensures that the mean trend in growth does not swamp the variance. If the mean were of larger order, it would dominate, and the variance would not be of much importance. Thus, this order assumption for the mean restricts the analysis to situations where the variance will be of significance to the results. As the growth rate of the invader in the twospecies SLM is mathematically equivalent to this age-structured density-independent model, this approximation (14) can be used to analyze the SLM.

\subsubsection{Nonstructured lottery model (NLM) and storage effect}

The nonstructured lottery model (NLM), which was originally developed to model coral reef fish communities (Chesson and Warner, 1981), has been studied and used for various purposes in a number of modified forms.

For the purpose of understanding the more general theoretical concept, the storage effect (Chesson, 1989, 1994; Chesson and Huntly, 1997), the NLM has served well because of its simplicity. Coexistence by the storage effect involves interactions between the response of population growth to competition, and the response to the varying environment. As we shall see below, this interaction can be measured by a single quantity $\gamma$, and this quantity can arise from differences in the sensitivities of different stages in the life cycle to environmental 
and competitive factors (Chesson, 1990). In both the NLM and SLM, sensitivities to environment and competition differ between the juvenile and adult stages. Production of juveniles is sensitive to the environment, and their survival to adulthood is sensitive to competition. In contrast, adult survival is assumed insensitive to both environmental and competitive factors.

The effect of a varying environment is represented in the lottery model by the varying birth rates, $b_{i}(t)$. The environmental response $E_{i}(t)$ is defined as the natural $\log$ of $b_{i}(t)$, i.e. $E_{i}(t)=\ln b_{i}(t)$, and is simply a more useful way of parameterizing the lottery model to determine the effect of environmental fluctuations. The magnitude of competition can be quantified as the number of juveniles competing relative to the amount of space that they are competiting for. In the residentinvader scenario developed above, residents are solely responsible for competition, and the density of space they give up is $\tilde{\delta}_{j}$. The density of juveniles competing for this space is simply $b_{j}(t)$. Thus, the magnitude of competition is $b_{j}(t) / \tilde{\delta}_{j}$. Taking logs of this, gives the competitive response $C=\ln b_{j}(t) / \tilde{\delta}_{j}$. With these definitions, the growth rate of the resident species $j$ takes the form

$r_{j}(t)=\ln \left\{\left(1-\tilde{\delta}_{j}\right)+e^{E_{j}-C}\right\}$.

The growth rate of the invader is slightly more complicated, because the age structure of the invader does fluctuate over time. Nevertheless, the invader growth rate becomes

$r_{i}(t)=\ln \left\{\left(1-\hat{\delta}_{i}(t)\right)+\hat{k_{i}}(t) e^{E_{i}-C}\right\}$

differing only from the NLM in the presence of $\hat{k_{i}}$ and the deviation of $\hat{\delta}_{i}(t)$ from $\tilde{\delta}_{i}$.

To analyze the model, we introduce the quantity $\mu$ which characterizes the dynamics of the system under constant environmental conditions:

$\mu=E \ln \frac{b_{i}(t)}{\tilde{\delta}_{i}}-E \ln \frac{b_{j}(t)}{\tilde{\delta}_{j}}$.

If the environment does not fluctuate over time and with the quantities $E_{i}(t)$ and $E_{j}(t)$ fixed at their expected values, the sign of $\mu$ determines which species will ultimately dominate the system. If $\mu$ is positive, species $i$ will eliminate species $j$ from the system. This fact has long been known for the NLM (Chesson and Warner, 1981), and is not difficult to verify also for the SLM. In a variable environment, with species having the same mortality schedules and the same variances for $E_{i}$ and $E_{j}$, the long-term growth rate of the invader can be approximated as

$\bar{r}_{i}=\tilde{\delta} \mu+\Delta I$,

(Chesson, 1989, 1994) where the quantity $\Delta I$ is a term called the storage effect, which results from an interaction between fluctuations in the environment, competition and species. When positive, it can outweigh the effects of $\mu$ on the growth of the invader, permitting $\bar{r}_{i}$ to be positive for both species when invader, and allowing coexistence by the invasibility criterion.

In the nonstructured case with $\tilde{\delta}_{j}=\tilde{\delta}_{i}$, the storage effect satisfies the following formula:

$\Delta I=\gamma_{i} \operatorname{Cov}\left(E_{i}, C\right)-\gamma_{j} \operatorname{Cov}\left(E_{j}, C\right)$.

The quantity $\gamma_{i}$ is equal to the cross partial derivative of $r_{i}$ with respect to $E_{i}$ and $C$, which here reduces to $-\tilde{\delta}_{i}\left(1-\tilde{\delta}_{i}\right)$. This quantity is a measure of the nonadditivity of $r_{i}$ as a function of $E_{i}$ and $C$. Under the assumptions that we have made here (equal adult death rates $\tilde{\delta}$ for the two species, and equal variances, $\left.\operatorname{Var}\left(E_{i}\right)=\operatorname{Var}\left(E_{j}\right)\right)$, the storage effect reduces to the particularly simple form

$\tilde{\delta}(1-\tilde{\delta}) \sigma^{2}$,

where $\sigma^{2}=\frac{1}{2} \operatorname{Var}\left(E_{i}-E_{j}\right)$, i.e. half the variance of the difference in the $\ln b$ values of the two species. In this situation, formula (16) for $\bar{r}_{i}$ implies that both species have positive long-term growth rates as invaders, and hence coexist, when

$\sigma^{2}>\frac{|\mu|}{\left(1-\tilde{\delta}_{i}\right)}$,

for $i=1,2$. This approximation applies generally to the NLM for small variances of the $E_{i}$ and $E_{j}$. The variances do not need to be equal, $E_{i}$ and $E_{j}$ can be correlated arbitrarily, and the $\tilde{\delta}$ s may differ between species. However, when $\tilde{\delta}$ s do differ between species, Eq. (16) has an additional term $\Delta N$ (Chesson, 1994), which, however, still reduces to the same condition (18) when combined with $\Delta I$ in the NLM (Chesson, 1989).

In the SLM, the resident species $j$ has an identical equation for $r_{j}(t)$ as the NLM, and so its nonadditivity, $\gamma$, and covariance are unchanged. The invading species, however, differs in that $\hat{\delta}_{i}(t)$ and $\hat{k}_{i}$ fluctuate over time, but $\operatorname{Cov}\left(E_{i}, C\right)$ is unaffected. Thus, there are some deviations of the results of the SLM from the simple formulae above, which we now investigate using the $\Delta$ measures.

\subsection{The SLM with structured mortality only}

First, let us consider only the addition of structured mortality to the model. Thus, $k_{i x}=k_{j x}=1$ for all $x$, and Eq. (7) for the invader becomes

$P_{i .}(t+1)=P_{i .}(t)\left\{1-\sum_{x=1}^{\infty} \delta_{i x} u_{i x}(t)+\tilde{\delta}_{j} \frac{b_{i}(t)}{b_{j}(t)}\right\}$. 
The long-term population growth rate of the invader is

$$
\begin{aligned}
\bar{r}_{i} & =E\left[\ln \left\{\frac{P_{i .}(t+1)}{P_{i .}(t)}\right\}\right] \\
& =E\left[\ln \left\{1-\sum_{x=1}^{\infty} \delta_{i x} u_{i x}(t)+\tilde{\delta}_{j} \frac{b_{i}(t)}{b_{j}(t)}\right\}\right] \\
& =E\left[\ln \left\{1-\hat{\delta}_{i}(t)+\tilde{\delta}_{j} \frac{b_{i}(t)}{b_{j}(t)}\right\}\right],
\end{aligned}
$$

where the average death rate of the invader over age classes, which now is time dependent due to temporal fluctuations in the age distribution, is

$\hat{\delta}_{i}(t)=\sum_{x=1}^{\infty} \delta_{i x} u_{i x}(t)$

By assuming that fluctuations in the age distribution are not important (justified in the cases where species are long-lived) and assuming that $\bar{r}$ is small, Dewi and Chesson (2003) show that the average over age classes of the death rate of the invader $\left(\hat{\delta}_{i}(t)\right)$ can be expressed as

$\hat{\delta}_{i}(t) \approx \tilde{\delta}_{i}\left(1-\bar{r}_{i} \Delta_{m}\right)$,

where

$\Delta_{m}=\sum_{x=1}^{\infty} x l_{i x}\left(\delta_{i x}-\tilde{\delta}_{i}\right)$

All three types of mortality schedule will be investigated for the simple case where we make a distinction only between old and young adults, with constant death rates within these two groups. This situation leads to simple algebra while still exhibiting important effects of age-dependence of death rates. For the type II mortality schedule, the death rate $\left(\delta_{x}\right)$ is the same for all ages, and equal to $\tilde{\delta}$. For a type I mortality schedule

$\delta_{x}= \begin{cases}\delta_{1}<\tilde{\delta} & \text { if } x<s \\ \delta_{2}>\tilde{\delta} & \text { otherwise }\end{cases}$

where $s$ is the age at which the death rate changes from the early mortality rate $\left(\delta_{1}\right)$ to the late mortality rate $\left(\delta_{2}\right)$. A type III mortality schedule is opposite to a type I mortality schedule in that mortality is higher in the younger age classes.

In comparing the performance of the NLM and the SLM, we fix the age-independent death rate $(\tilde{\delta})$. This fixes the expected lifetime $(1 / \tilde{\delta})$, and focuses attention on the shape of the mortality schedule rather than the overall mortality level or longevity. Given the early mortality rate $\left(\delta_{1}\right)$, and the age, $s$, at which the death rate changes from the early rate to the late mortality, the mortality schedule can be expressed as

$\delta_{x}= \begin{cases}\delta_{1} & \text { if } x<s, \\ \frac{\left(1-\delta_{1}\right)^{s-1} \delta_{1}}{\left(\delta_{1} / \tilde{\delta}\right)-1+\left(1-\delta_{1}\right)^{s-1}} & \text { otherwise. }\end{cases}$
We then use $\Delta_{m}$ as defined by (23) to quantify the deviation of this mortality schedule from type II, and see how this deviation affects the behavior of the SLM in the sections below.

\subsection{The SLM with structured mortality and fecundity}

Now we include structured fecundity in our examination of the SLM. Modulation of reproduction ( $k$ function) will take the most common shapes: uniform, asymptotic, and triangular (see Fig. 2 and Roff, 1992), as discussed above.

Eq. (7) implies that the long-term population growth rate of the invader is

$$
\begin{aligned}
\bar{r}_{i} & =E\left[\ln \left\{1-\sum_{x=1}^{\infty} \delta_{i x} u_{i x}(t)+\tilde{\delta}_{j} \frac{b_{i}(t)}{b_{j}(t)} \sum_{x=1}^{\infty} k_{i x} u_{i x}(t)\right\}\right] \\
& =E\left[\ln \left\{1-\hat{\delta}_{i}(t)+\tilde{\delta}_{j} \frac{b_{i}(t) \hat{k_{i}}(t)}{b_{j}(t)}\right\}\right]
\end{aligned}
$$

where $\hat{\delta}_{i}(t)$ is as in Eq. (21), and the average modulation of reproduction of the invader over age classes is

$\hat{k_{i}}(t)=\sum_{x=1}^{\infty} k_{i x} u_{i x}(t)$.

By applying $\Delta_{f}$, again in the case where high longevity permits fluctuations in age structure to be ignored (Dewi and Chesson, 2003), we can express the average modulation of reproduction over age classes as

$$
\begin{aligned}
\hat{k_{i}}(t) & \approx \tilde{k_{i}}(t)\left(1+\bar{r}_{i} \Delta_{f}\right) \\
& =1+\bar{r}_{i} \Delta_{f},
\end{aligned}
$$

where

$\Delta_{f}=\tilde{\delta} \sum_{x=1}^{\infty} x l_{i x}\left(k_{i x}-1\right)$.

The relationship of $\Delta_{f}$ to the different types of fecundity schedules was given by (12). These fecundity schedules are classified in terms of the age at which the majority of offspring are produced, rather than the age at which reproduction begins.

\subsubsection{The long-term population growth rate of the invader and the storage effect}

We will now examine the long-term population growth rate of the invader with structured mortality and fecundity, given in Eq. (24), by considering the equation for the long-term population growth rate of the invader in the SLM as a special case of the population growth rate of the stochastic demographic model from Dewi and Chesson (2003) discussed above in the section on $\Delta$-measures. The birth rate $\tilde{b}(t)$ used there corresponds by Eqs. (7) and (8) to $\tilde{\delta}_{j} b_{i}(t) / b_{j}(t)$. It 
follows that the corresponding definition of $\mu$ here is

$\mu=E \ln \frac{b_{i}(t)}{\tilde{\delta}_{i}}-E \ln \frac{b_{j}(t)}{\tilde{\delta}_{j}}$,

which agrees with the definition of $\mu$ for the NLM, Eq. (15).

To obtain an expression for the long-term growth rate, we note that we assumed above that $\operatorname{Var}\left[\ln b_{i}(t)\right]=$ $\operatorname{Var}\left[\ln b_{j}(t)\right]=\sigma^{2}$, and that $b_{i}(t)$ and $b_{j}(t)$ are independent. These are merely assumptions for convenience of the simulations because the dynamics of the system depend only on $\operatorname{Var}\left[\ln b_{i}(t)-\ln b_{j}(t)\right]$, which equals $2 \sigma^{2}$ under the assumptions in force here. Moreover, here $\sigma^{2}$ is equivalent to $\frac{1}{2} \sigma^{2}$ in the demographic model in Dewi and Chesson (2003) summarized in the subsection on $\Delta$-measures above. Applying result (14), above, noting this difference in the definition of $\sigma^{2}$, we obtain

$\bar{r}_{i}=\frac{\tilde{\delta}_{i}\left[\mu+\left(1-\tilde{\delta}_{i}\right) \sigma^{2}\right]}{1+\tilde{\delta}_{i}\left(\Delta_{f}-\Delta_{m}\right)}+o\left(\sigma^{2}\right)$.

For coexistence, this value must be positive for each species as an invader, which means that coexistence occurs when

$\sigma^{2}>\frac{|\mu|}{\left(1-\tilde{\delta}_{i}\right)}$,

for each species (i.e. $i=1,2$ ) which agrees with the coexistence criterion for the NLM (expression (18)). According to this formula, the details of the mortality and fecundity schedules do not affect species coexistence.

The component of Eq. (28) that reflects fluctuations, i.e. the storage effect component, is

$\frac{\tilde{\delta}_{i}\left(1-\tilde{\delta}_{i}\right) \sigma^{2}}{1+\tilde{\delta}_{i}\left(\Delta_{f}-\Delta_{m}\right)}$,

which reduces, as it should, to the storage effect in the NLM (expression (17)) when $\Delta_{m}=0$ and $\Delta_{f}=0$. When the mortality schedule is of type I, or the fecundity schedule is early peak reproduction, the strength of the storage effect is increased. Indeed, all components of $\bar{r}_{i}$ are increased in these situations, and are decreased when mortality is of type III or fecundity is delayed peak. Thus, the effects of age structure are approximately to speed or slow the long-term growth of an invader uniformly, but not to affect its sign or interact appreciably with the coexistence mechanism here, the storage effect.

\section{Simulation results}

The expressions for $\bar{r}_{i}$ above are approximate, and have some restrictions whose effects are not fully understood. Here we present results from simulations exploring the long-term population growth rate of the invader, and mean values of $\hat{\delta}_{i}$ and $\hat{k_{i}}$ from Eqs. (21) and (25), respectively. For the simulations, we consider only the symmetric case where the two species have the same mortality schedules and the same modulation of fecundity by age. We assume throughout that the expected lifetime is equal to 9 , and impose the usual constraint (Eq. (1)) that the population average of $k_{x}$ for a stationary population is equal to 1 . In the invasibility analyses, the invader species is chosen to be the species with lower mean birth rate, and is denoted by the subscript $i$. The resident species, denoted by the subscript $j$, is always at its stationary distribution. A species with higher mean birth rate than the resident (or more generally, a higher value of $\mu$ ) will always be able to invade, given that all other parameters are equal. Thus, disadvantaged invaders are our focus, since if a disadvantaged species can invade, it will coexist with its competitor.

Some of the mortality and fecundity schedules to be used in the simulations are presented in Figs. 1 and 2. It can be seen from Fig. 1 that the larger the magnitude of $\left|\Delta_{m}\right|$, the more the mortality schedule departs from a type II schedule. The larger the magnitude of $\left|\Delta_{f}\right|$, the more the fecundity schedule deviates from age-independent reproduction, even though the classification of fecundity schedules is less distinct than that of mortality schedules. Age-independent reproduction means that the age of mean reproduction is equal to the expected lifetime (i.e., equal to 9 in our case). Fig. 2 shows that, with early peak reproduction, more than half of the area under the fecundity modulation curve lies before $x=9$, meaning that the majority of offspring are produced early in life. With the delayed peak reproduction, less than half of the area lies before $x=9$, meaning that the majority of offspring are produced later in life, given survival to old age. Fig. 2 also shows that the case of delayed peak reproduction, the fecundity curve with the highest $\Delta_{f}$ value, viz. $\Delta_{f}=7.1594$, has the smallest area under the fecundity curve before age $x=9$.

When structured mortality and fecundity are combined, the mean age of reproduction is shifted according to the mortality schedule (Fig. 3). A type I mortality schedule will shifts the age of mean reproduction for age-independent reproduction to the right (later age). The opposite shift occurs with a type III mortality schedule.

Differences between the mean of $\ln$ birth rates for the invader and the resident $(\mu)$ in simulations range from -0.4 to -0.1 . Variances of $\ln$ birth rates for the invader and the resident are kept equal, and the common variances $\left(\sigma^{2}\right)$ range from 0 to 0.5 .

\subsection{The long-term population growth rate of the invader}

First, let us compare the simulation results for the long-term population growth rate of the invader when 

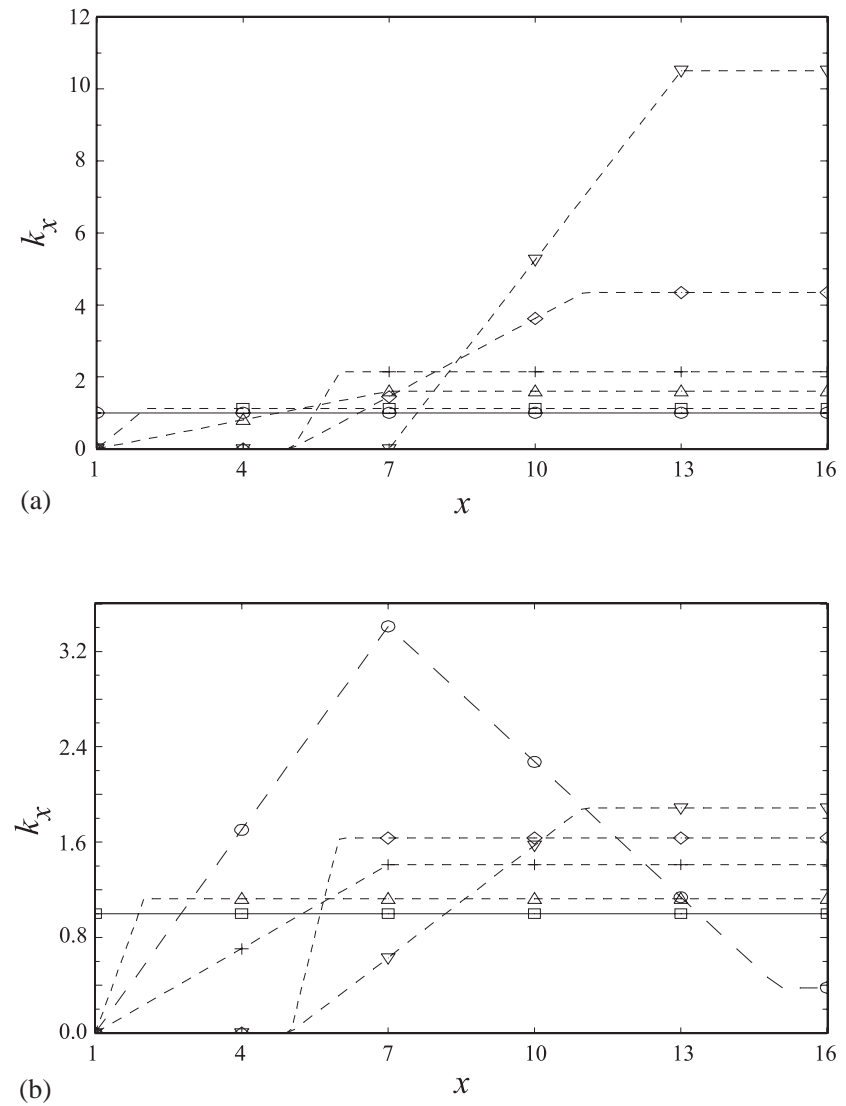

Fig. 3. A type I mortality schedule with $\Delta_{m}=0.4086$, with ageindependent reproduction $(\circ)$, and delayed peak reproduction, with $\Delta_{f}=0.5399(\square), \Delta_{f}=1.6264(\triangle), \Delta_{f}=2.7015(+), \Delta_{f}=$ $3.5373(\diamond)$, and $\Delta_{f}=4.5645(\nabla)($ a). A type III mortality schedule with $\Delta_{m}=-1.9485$, early peak reproduction, with $\Delta_{f}=-12.2130(\circ)$, age-independent reproduction $(\square)$, and delayed peak reproduction, with $\Delta_{f}=3.1922(\triangle), \Delta_{f}=9.9659(+), \Delta_{f}=15.1978(\diamond)$, and $\Delta_{f}=20.5213(\nabla)(\mathrm{b})$.

mortality and/or fecundity are structured (the SLM), with these when mortality is of type II and reproduction is independent of age (the NLM) (Figs. 4, 6, and 7).

We will first consider the SLM with structured mortality only (Fig. 4). When the invader population is decreasing, the long-term population growth rate of the invader is lower with a type I mortality schedule than with a type II mortality schedule. When the population is increasing, the long-term population growth rate is higher with a type I than a type II mortality schedule (Fig. 4(a)). In other words, when an invader is recovering from low density, an invader with a type I mortality schedule will recover faster than an invader with a type II mortality schedule. However, when the population is declining, a type I invader will decline faster than a type II invader. With a type III mortality schedule (Fig. 4(b)), the recovery rate of a type III invader is lower than that of type II invader (and therefore, a type I invader), and the extinction rate is also lower than that of types I and II invaders. Although
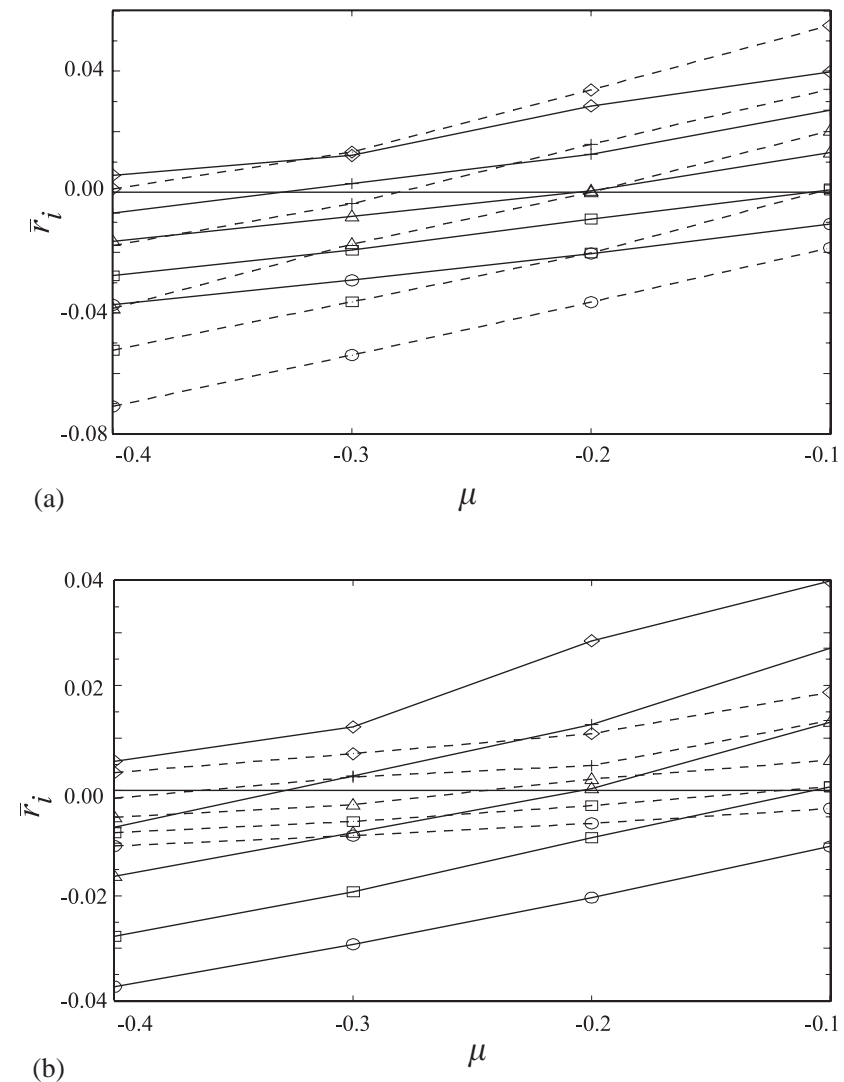

Fig. 4. Long-term population growth rate of the invader $\left(\bar{r}_{i}\right)$ calculated using simulations with mortality schedules of type II (solid lines) and type I with $\Delta_{m}=0.4086$ (dashes) (a), and type III with $\Delta_{m}=-1.9485$ (dashes) (b). $\sigma^{2}$ equals $0(\circ), 0.125(\square)$, $0.25(\triangle), 0.375(+)$, and $0.5(\diamond)$.

the simulations were done with residents having the same mortality schedule as the invader, the age-structure of the resident is stationary, and so the shape of its mortality schedule has no effect on the invader growth rate. Thus, this comparison of the effects of invader mortality schedules is independent of the mortality schedules of the residents with which they compete.

The fecundity schedules behave similarly to the mortality schedules (Fig. 6) in their effects on the recovery rate of the invader. Early peak reproduction behaves like a type I mortality schedule, i.e., increases $\left|\bar{r}_{i}\right|$, and delayed peak reproduction behaves like a type III mortality schedule, i.e., decreases $\left|\bar{r}_{i}\right|$.

Now let us look at how mortality and fecundity schedules affect the coexistence criterion, i.e., the magnitude of $\sigma^{2}$ required for the invader to have a zero long-term population growth rate. Looking closely at the horizontal line $\left(\bar{r}_{i}=0\right)$ in Figs. 4(a) and (b), the intersection of type I is to the right of that of type II which is to the right of that of type III. This pattern is less obvious with smaller $\sigma^{2}$. The pattern shows that, in order to persist, a type I invader requires larger $\sigma^{2}$ than a type II invader, which requires larger $\sigma^{2}$ than a type III 

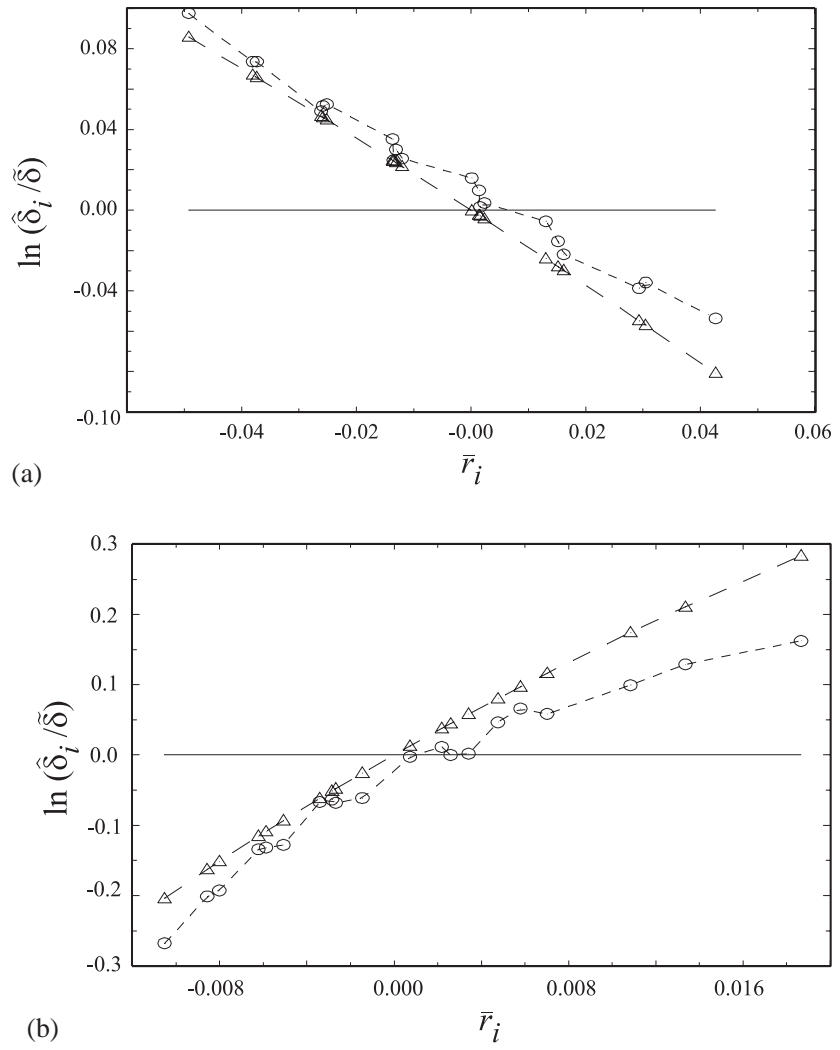

Fig. 5. The ln of ratio of mean value of $\hat{\delta}_{i}(t)$ from equation (21), calculated using simulation, and age-independent death rate $(\tilde{\delta})(\circ)$, and the $\ln$ of ratio of $\hat{\delta}_{i}$, from equation (22), and age-independent death rate $(\tilde{\delta})(\triangle)$, with a type I mortality schedule with $\Delta_{m}=0.2025$ (a) and a type III mortality schedule with $\Delta_{m}=-1.9485$ (b).

invader with the same level of inferiority $(\mu)$, even though little quantitative difference between the three can be seen in the figures.

The small quantitative effect of structured mortality on coexistence is consistent with the assumption used in deriving approximation (22) that stochastic fluctuations in age distribution have negligible effect on the overall adult mortality rate of the invading population. According to Eq. (22), the average death rate of the invader over age classes $\left(\hat{\delta}_{i}(t)\right)$ should be approximately equal to $\tilde{\delta}_{i}$ when $\bar{r}_{i}=0$. However, Fig. 5 shows that there are some small discrepancies between the mean values of $\hat{\delta}_{i}$ from Eq. (21), calculated using simulation, and $\tilde{\delta}$. With a type I mortality schedule (Fig. 5(a)), the mean value of the average death rate of the invader over age classes $\left(\hat{\delta}_{i}\right)$ calculated using simulation is slightly larger than the age-independent death rate $(\tilde{\delta})$ when the long-term population growth rate of the invader $\left(\bar{r}_{i}\right)$ is equal to zero. The opposite pattern occurs with a type III mortality schedule, i.e., the mean of $\hat{\delta}_{i}$ calculated using simulation is slightly smaller than $\tilde{\delta}$ when $\bar{r}_{i}=0$. This nonzero $\ln \left\{\hat{\delta}_{i}(t) / \tilde{\delta}_{i}\right\}$ when $\bar{r}_{i}=0$ appears to be responsible for the small quantitative effect of structured mortality on coexistence.
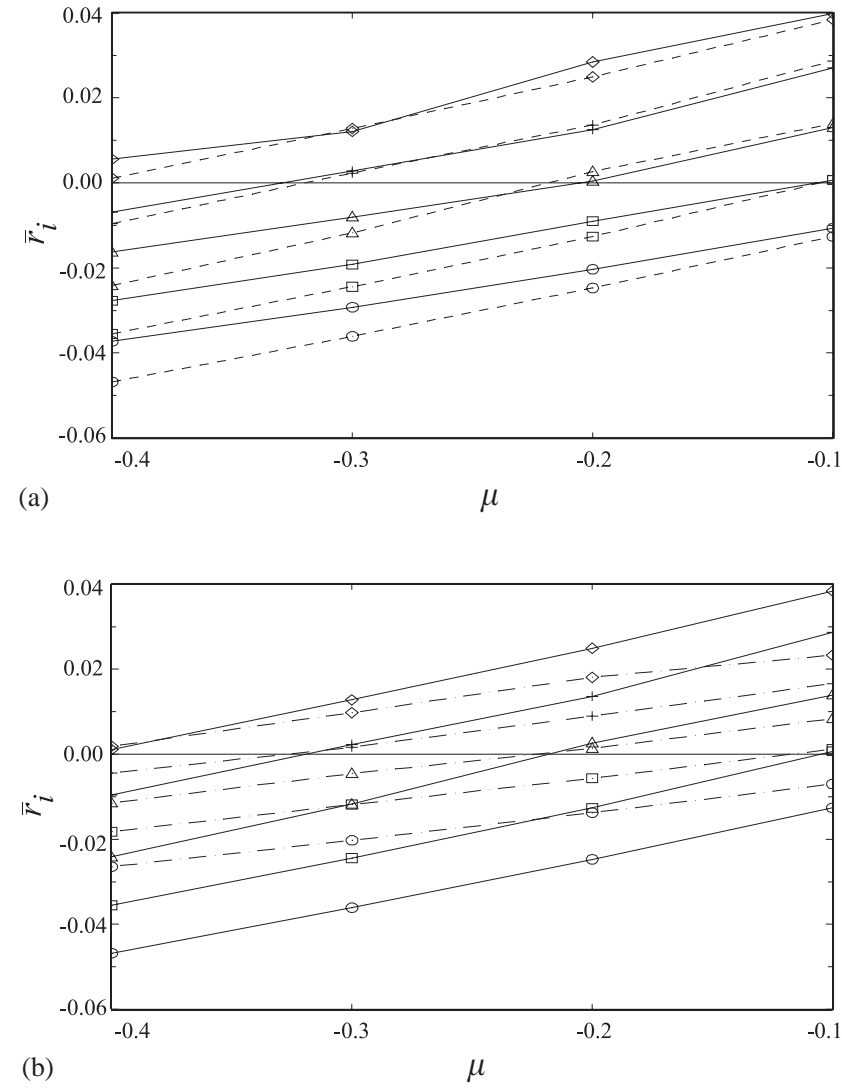

Fig. 6. Long-term population growth rate of the invader $\left(\bar{r}_{i}\right)$, calculated using simulations, with mortality schedule of type II and age-independent reproduction (solid lines), and with an early peak reproduction with $\Delta_{f}=-1.2545$ (short dashes) (a), and with a delayed peak reproduction with $\Delta_{f}=5$ (long dashes) (b). $\sigma^{2}$ equals $0(\circ), 0.125(\square), 0.25(\triangle), 0.375(+)$, and $0.5(\diamond)$.

In Figs. 6 and 7 there are no apparent differences in the location of the intercept $\bar{r}_{i}=0$ between agedependent and age-independent fecundity schedules. Thus, it appears that coexistence is not appreciably affected by fecundity schedules. The mean value of the average modulation of reproduction of the invader over age classes $\left(\hat{k}_{i}(t)\right)$ from Eq. (25), calculated using simulation, is very close to one (Fig. 8), when the long-term population growth rate of the invader $\left(\bar{r}_{i}\right)$ is zero. This explains why there is no appreciable effect of structured fecundity on coexistence.

The invader with early peak reproduction has the mean value of $\hat{k}_{i}$ less than 1 when the population is decreasing, and greater than 1 when the population is increasing. The opposite pattern occurs for delayed peak reproduction. This qualitative pattern of the mean values of $\hat{k_{i}}$ is found to be consistent throughout all combinations of fecundity schedules and mortality schedules in the parameter set.

Approximation of the long-term population growth rate of the invader, given by Eq. (28), works well, both qualitatively and quantitatively in all cases: (i) with 


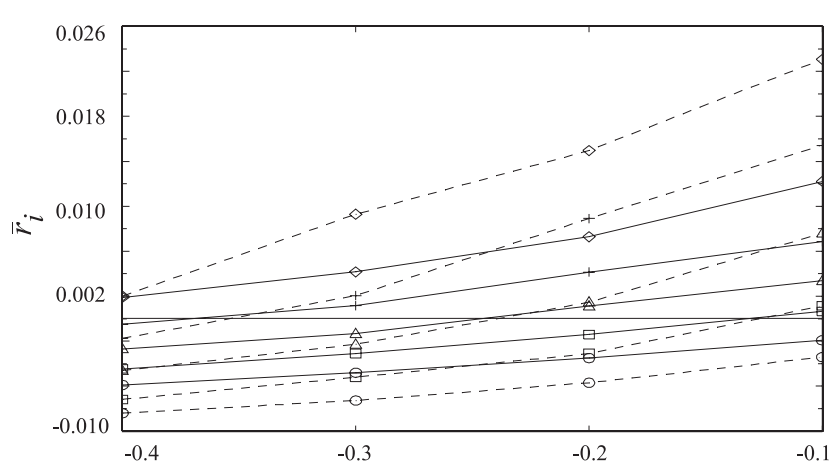

(a)

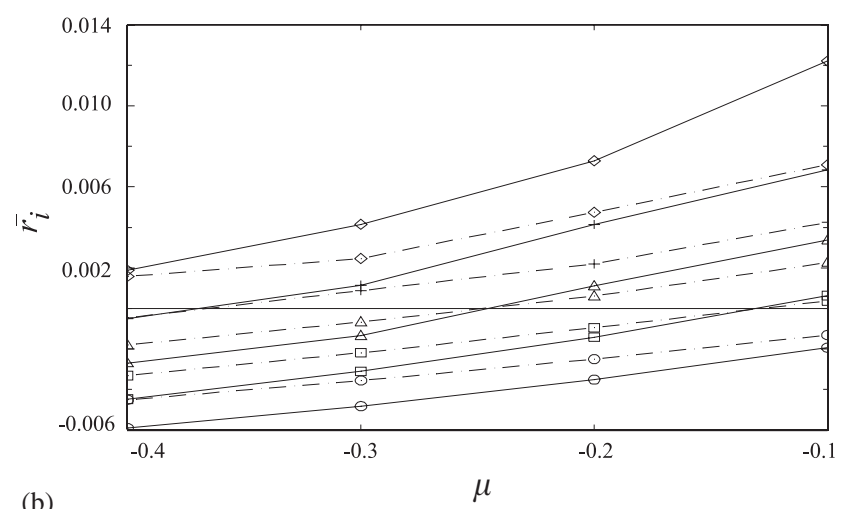

(b)

Fig. 7. Long-term population growth rate of the invader $\left(\bar{r}_{i}\right)$, calculated using simulations, with mortality schedule of type III with $\Delta_{m}=-4.1589$ and age-independent reproduction (solid lines), and with an early peak reproduction with $\Delta_{f}=-22.6039$ short dashes) (a), and with a delayed peak reproduction with $\Delta_{f}=25.3683$ (long dashes) (b). $\sigma^{2}$ equals $0(\circ), 0.125(\square), 0.25(\triangle), 0.375(+)$, and $0.5(\diamond)$.

structured mortality (Fig. 9(a)), (ii) with structured fecundity (Fig. 9(b)), and (iii) with structured mortality and structured fecundity (Fig. 10(b)).

\section{Discussion}

We have extended the non-structured lottery model (NLM) to an age-structured lottery model (SLM) with age-dependent mortality and fecundity in the adult stage. Competition for space occurs only in the juvenile stage in both models. The life-history parameters defining the NLM (Chesson and Warner, 1981; Hatfield and Chesson, 1989; Chesson, 1990, 1994) are the adult mortality rates and the birth rates for each species. In general, both mortality rates and birth rates can fluctuate over time, but we consider here only fluctuating birth rates, as only their fluctuations promote coexistence. Note that the expected lifetime is just the reciprocal of the adult mortality rate.

To compare the NLM and SLM intrinsically, we imposed the natural constraints of fixing the expected lifetime, while varying the age-specific mortality rates in
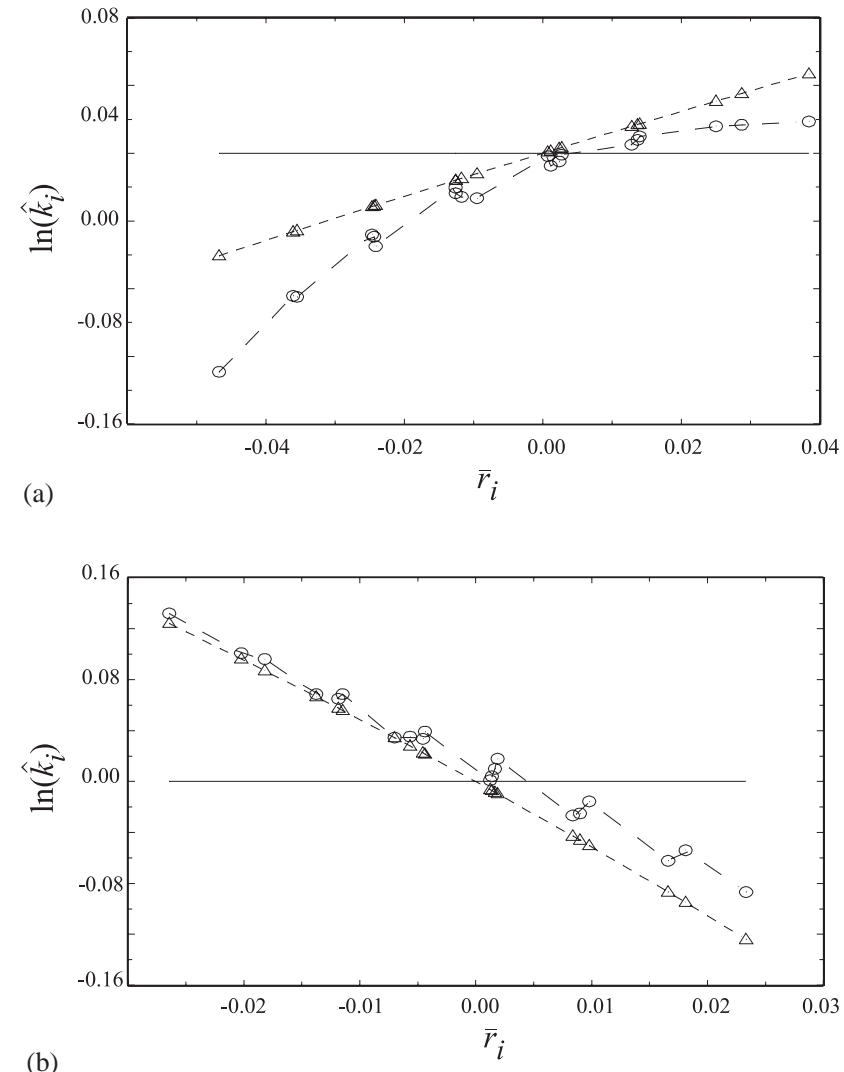

(b)

Fig. 8. The $\ln$ of mean value of $\hat{k_{i}}(t)$ from equation (25), calculated using simulation $(\circ)$, and $\ln \hat{k}_{i}$ from equation (26) $(\triangle)$, with an early peak reproduction with $\Delta_{f}=-1.2545$ (a), and a delayed peak reproduction with $\Delta_{f}=5$ (b).

the SLM, and by varying the age-specific birth rates in such a way that the population-level birth rate (the number of births divided by the number of individuals), at its stationary age structure, remains the same. Note that this population-level birth rate at the stationary age structure is simply equal to the net reproductive rate of demographic theory (Caswell, 2001) divided by the expected lifetime. Thus, the SLM and NLM are being compared for equal expected lifetimes and equal net reproductive rates. The population level, effects of age structure, however, are captured in the quantities $(\hat{\delta})$ and $(b \hat{k})$ which are defined as the death rates and birth rates at the population level, given the actual agestructure found at that time. These quantities may deviate from the values $\tilde{\delta}$ and $b$ in the comparable NLM whenever the age structure differs from the stationary age structure, and these deviations cause the NLM and SLM to differ in their dynamics and long-term lowdensity growth rates. In an resident-invader situation, however, the resident is always at its stationary age structure in the SLM. The invader is nonstationary in general, and so the population-level mortality rates and birth rates differ from their stationary values. These 

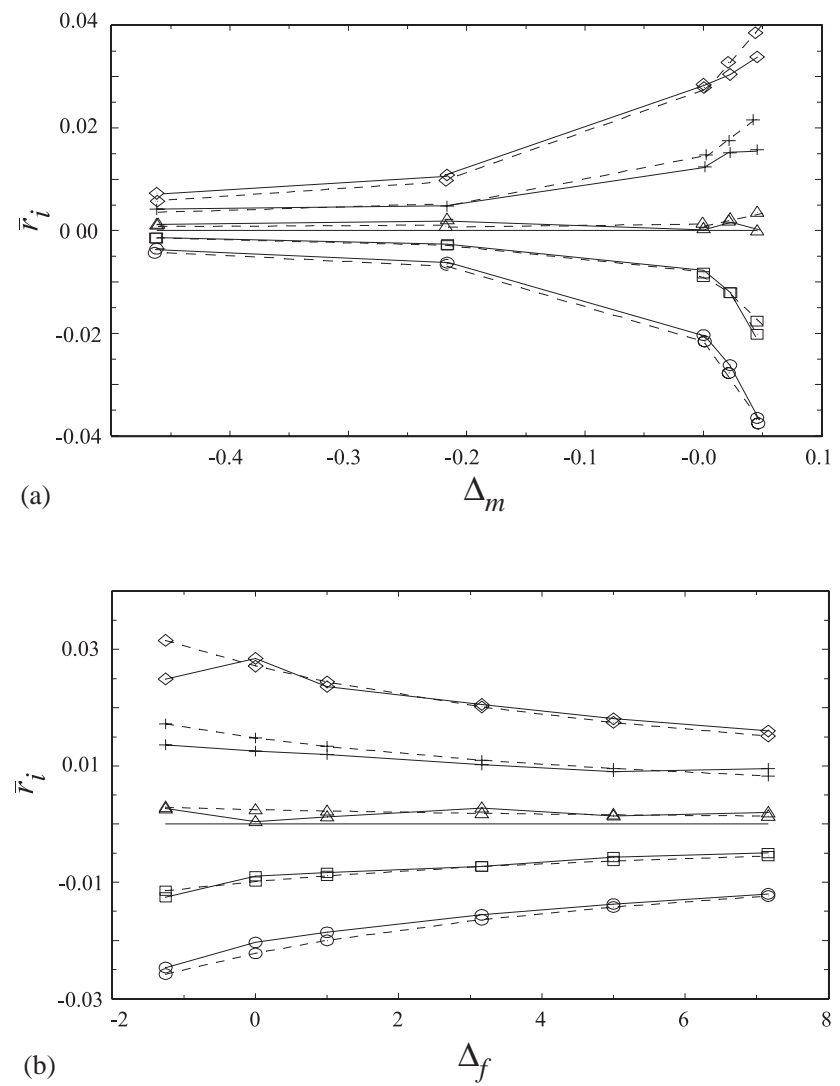

Fig. 9. Long-term population growth rate of the invader $\left(\bar{r}_{i}\right)$ with various mortality schedules and age-independent reproduction, calculated using simulation (solid), and equation (28) (dashes) (a). Longterm population growth rate of the invader $\left(\bar{r}_{i}\right)$ with various fecundity schedules and a type II mortality schedule, calculated using simulations (solid), and equation (28) (dashes) (b). $\sigma^{2}$ equals $0(\circ)$, $0.125(\square), 0.25(\triangle), 0.375(+)$, and $0.5(\diamond) . \mu=-0.2$.

effects on the invader, but not on the resident, in the SLM, explain the effects of mortality and fecundity schedules on the long-term population growth rate of the invader.

A type III mortality schedule results in lower population average mortality rates in declining populations, and higher population average mortality rates in increasing populations. A type I mortality schedule results in higher population average mortality rates in declining populations, and lower population average mortality rates in increasing populations. Delayed peak reproduction has the same effect on the population average birth rates as does a type III mortality schedule, i.e., it results in higher population average birth rates when populations are decreasing, and lower population average birth rates when populations are increasing. Early peak reproduction behaves in the same way as a type I mortality schedule. These effects are responsible for changes in the long-term low-density growth rate of the invader as mortality and fecundity schedules are varied.
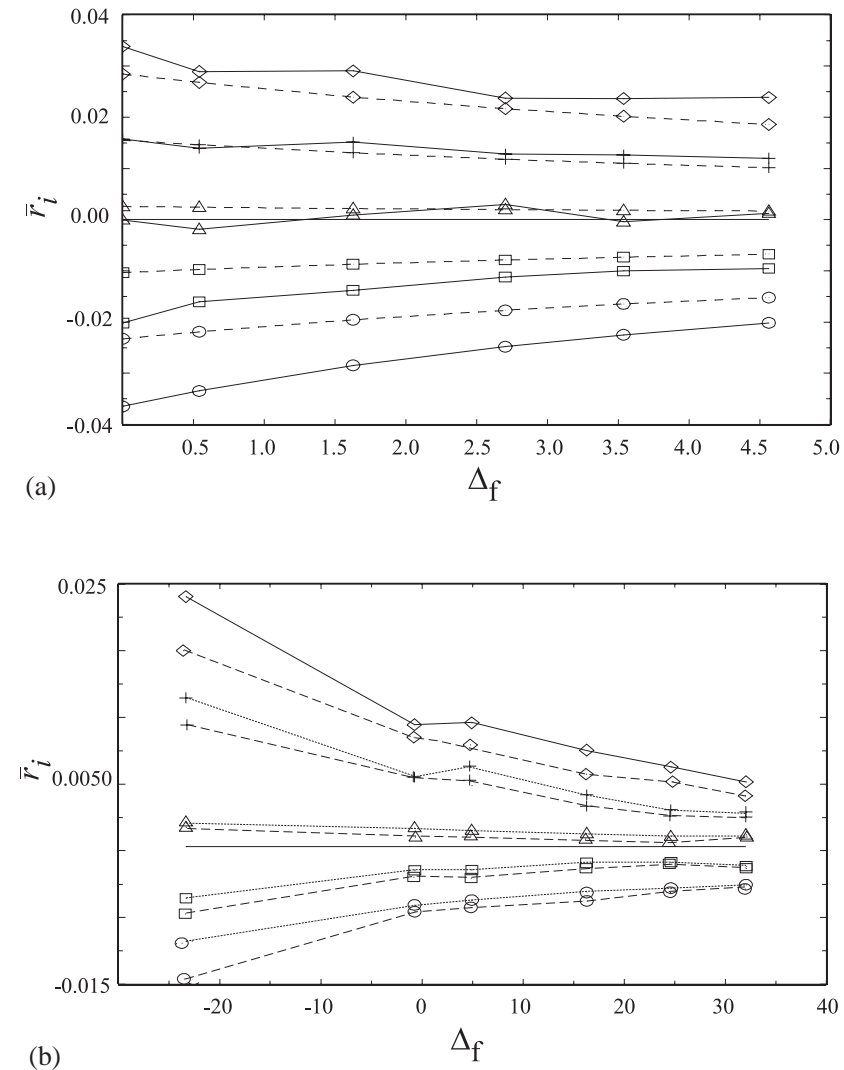

Fig. 10. Long-term population growth rate of the invader $\left(\bar{r}_{i}\right)$ with various fecundity schedules and a type I mortality schedule with $\Delta_{m}=$ 0.4086 , calculated using simulations (solid), and equation (28) (dashes) (a). Long-term population growth rate of the invader $\left(\bar{r}_{i}\right)$ with various fecundity schedules and a type III mortality schedule with $\Delta_{m}=$ -1.9485 calculated using simulations (solid), and equation (28) (dashes) (b). $\sigma^{2}$ equals $0(\circ), 0.125(\square), 0.25(\triangle), 0.375(+)$, and $0.5(\diamond) . \mu=-0.2$.

The conditions for coexistence, as determined by simulation, do change with changes in mortality schedules, but quantitatively the changes in the coexistence conditions are small. These changes can only be due to fluctuations in the population age structure of the invader over time, because the coexistence boundary is defined by a zero long-term low-density growth rate, which implies that the age structure is the stationary age-structure, in agreement with the NLM, unless agestructure fluctuates over time. We found here that a type III mortality schedule always results in a less restrictive coexistence criterion than a type I mortality schedule. These changes may potentially be significant if for some reason the age structure of the invader fluctuates greatly over time.

It is unnecessary to perform simulation experiments using asymmetrical mortality and fecundity schedules for the two species because the resident is always at its stationary age structure and so the details of its mortality and fecundity schedules are unimportant. Thus, the effects of asymmetry between species in 
mortality and fecundity schedules can be deduced from the symmetrical simulations performed here.

Our analytical approximations for the long-term population growth rate of the invader reproduce the qualitative results of the simulation study, with the exception of the small effect of age structure on the coexistence region, because these approximations do not take into account the effects of fluctuating age structure. These approximations show how this longterm population growth rate and the storage effect depend on structured fecundity and mortality via the $\Delta$ measures quantifying the shapes of mortality and fecundity schedules. These results imply that the NLM and SLM behave similarly, with the primary effect of age-specific mortality and fecundity being to speed or slow growth from low density, without much effect on the sign of the growth rate, and hence on the conditions for coexistence. Evaluated at the stationary age-structure, the important components of the storage effect, viz covariance between environment and competition, and subadditivity, do not differ between the NLM and the SLM. These results suggest that use of the NLM is adequate for most theoretical purposes even though age-dependence might suggest use of the SLM. The approximations using the $\Delta$-measures indicate the kinds of situations where accounting for age-structure might be useful, and when these suggest large effects, simulation might be considered for more accurate results.

\section{Acknowledgments}

We are grateful for comments on an earlier version of this from Stephen Ellner and Hugh Possingham.
Support was provided in part by an AusAid Scholarship to Sonya Dewi and NSF grant DEB-0129833 to Peter Chesson.

\section{References}

Caswell, H., 2001. Matrix Population Models: Construction, Analysis, and Interpretation. Sinauer Associates, Inc. Publishers, Sunderland, MA.

Chesson, P.L., 1989. A general model of the role of environmental variability in communities of competing species. Lect. Math. Life Sci. 20, 97-123.

Chesson, P.L., 1990. Geometry, heterogeneity and competition in variable environments. Philos. Trans. R. Soc. London B 330, $165-173$

Chesson, P., 1994. Multispecies competition in variable environments. Theor. Popul. Biol. 45, 227-276.

Chesson, P.L., Ellner, S., 1989. Invasibility and stochastic boundedness in monotonic competition models. J. Math. Biol. 27, 117-138.

Chesson, P., Huntly, N., 1997. The roles of harsh and fluctuations conditions in the dynamics of ecological communities. Am. Nat. $150,519-553$.

Chesson, P.L., Warner, R.R., 1981. Environmental variability promotes coexistence in lottery competitive systems. Am. Nat. 117, 923-943.

Dewi, S., Chesson, P., 2003. Age-structured population growth rates in constant and variable environments: a near equilibrium approach. Theor. Popul. Biol. In Press.

Ellner, S., 1989. Convergence to stationary distributions in two-species stochastic competition models. J. Math. Biol. 27, 451-462.

Hatfield, J.S., Chesson, P.L., 1989. Diffusion analysis and stationary distribution of the two-species lottery competition models. Theor. Popul. Biol. 36, 251-266.

Roff, D.A., 1992. The Evolution of Life Histories. Chapman and Hall, London.

Warner, R.R., Chesson, P.L., 1985. Coexistence mediated by recruitment fluctuations: a field guide to the storage effect. Am. Nat. 125, 769-787. 